\title{
Design of Open-circuited Stub Butterworth Low-pass Microstrip Line Filter
}

\author{
Wen-Bin Lin ${ }^{\mathrm{a} *}$, Kao-Feng Yarn ${ }^{\mathrm{b}}$, Fenq-Lin Jenq $^{\mathrm{c}}$ \\ a lawrence@mail.feu.edu.tw \\ ${ }^{a}$ Department of Electronic and Optoelectronic Application Engineering, Far East University, Taiwan 74448, ROC \\ ${ }^{b}$ Department of Aircraft Maintenance, Far East University, Taiwan 74448, ROC \\ ${ }^{c}$ Electronic and Optoelectronic System Research Laboratories, Industrial Technology Research Institute, Taiwan 310, ROC
}

\begin{abstract}
This article designs a novel $2.5 \mathrm{GHz}$ Butterworth low pass microstrip line filter (MLF) by PCB prototyping machine. We could realize the theory, implement, and application of MLF in the same time .we design and simulate MLF circuit by IE3D software package. We choose FR-4 glass fiber PCB to make the MLF. We measure the S parameter frequency response of MLF by vector network analyzer.
\end{abstract}

Keywords: Microstrip line, filter, PCB prototyping machine.

\section{Introduction}

In recent years, with the advancement of science and technology, the popularization of smart technology has affected the quality of communications. Therefore, the carrier rate of communications has been continuously improved to increase bandwidth. In contrast, with the continuous increase of carrier frequency, microstrip line circuits are widely used in high-frequency circuits. From the traditional lumped components into the field of discrete component microstrip lines, understand the corresponding high-efficiency circuits, and then by designing and manufacturing microstrip line filters. Establish high-frequency circuit design concepts and understand the characteristics of high-frequency passive components.

We added the traditional semi-lumped element type microstrip line low-pass filter to the quarterwavelength open-circuited stubs to improve the original semi-lumped element type microstrip line low-pass filter, and got a production at $2.5 \mathrm{GHz}$ low-pass filter. Among them, the use of two quarter wavelength opencircuited stubs with different components produces different zero points, which greatly improves the insertion loss and reflection loss characteristics of the passband and stopband of the low-pass filter, especially the transition bandwidth becomes steep. This microstrip circuit is designed and simulated with IE3D microwave circuit simulation software, and uses FR-4 glass fiber printed circuit board as a substrate to make real products. 
We design and manufacture microwave microstrip line components, and understand the principle, production and application of microwave microstrip line components.

\section{Theoretical Framework}

Due to the rapid development of wireless communication systems and wireless network systems, various types of radio frequency components with small size, high performance, low radiation loss, high reliability, and low cost have become the focus of development. Planar passive components such as microstrip lines and coplanar waveguides (CPW) are used in various high-frequency passive components and various communication systems because of their simple structure, low cost, good production, and good characteristics.

A microstrip line basically has two conductor layers, one of which is used as a ground layer, and the other layer is a circuit line required for layout. The two conductor layers are separated by a flat insulating dielectric substrate and provide the conduction space required for electromagnetic waves [2-5]. This transmission line can be made into a microwave integrated circuit (MIC) using printed circuit board technology or various etching techniques in the semiconductor manufacturing process. Figure 1 is a microstrip line architecture diagram, where $d$ is the thickness of the dielectric substrate, $w$ is the width of the microstrip line, $t$ is the thickness of the conductor, and $\mathcal{E}_{r}$ is the dielectric constant of the dielectric substrate.

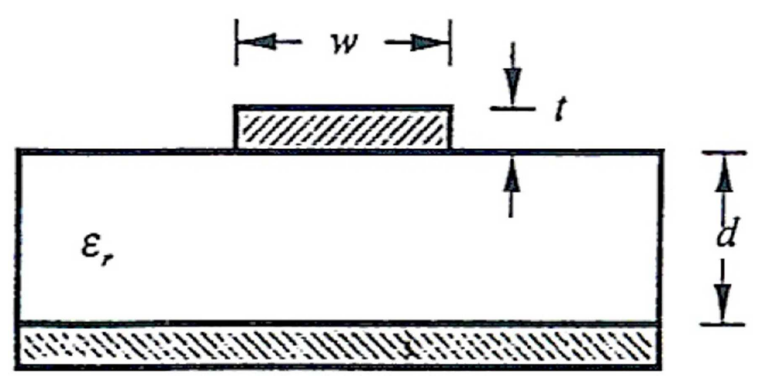

Figure 1. A microstrip line architecture diagram

General microstrip line production methods are as follows:

1. Thin film technology process: Due to the semiconductor process technology, the circuit quality and accuracy are high, but the process equipment and technology costs are high, the cost is high in small-scale production, and low in mass production.

2. Thick film technology process: The cost of process equipment and technology is lower than that of the semiconductor process, so when the output is medium, the cost is lower than that of the thin film technology process.

3. Printed circuit manufacturing process: The use of general printed circuit technology to produce microwave components or circuits is the simplest process and the accuracy is not high. However, the low cost in smallscale production is suitable for small-scale production and experimental use.

The dielectric constant and thickness of the dielectric substrate are two very important parameters in the circuit design of the microstrip line. The main job of the so-called circuit design is to calculate the physical dimension of the microstrip line, that is, the microstrip line. Wire width and wire length. A common microstrip line architecture diagram is shown in Figure 1 [1-11].

Although the accuracy of the printed circuit process is poor, considering the cost and ease of production, we think that the $2.5 \mathrm{GHz}$ microstrip line low-pass filter designed and produced is most suitable for the printed 
circuit board. By designing and manufacturing microstrip line filters, we can establish high-frequency circuit design concepts and understand the characteristics of high-frequency passive components to become familiar with the design and production process of microstrip line components and familiar with the application of engraving machines and microstrip components measuring.

\section{Research Methods and Steps}

We designed and fabricated a $2.5 \mathrm{GHz}$ microstrip line low-pass filter circuit for signal processing after frequency reduction in a $60 \mathrm{GHz}$ system on a printed circuit board, and discussed its characteristics. The research steps and methods are as follows:

We use IE3D microwave circuit simulation software to design the $2.5 \mathrm{GHz}$ microstrip line low-pass filter circuit we need. S-parameter frequency response graph obtained by simulation to confirm its circuit characteristics.

Figure 2 shows an example of my practice, using IE3D microwave circuit simulation software to simulate a traditional stepped-impedance low-pass filter (Stepped-Impedance Low Pass Filter), operating frequency 2.5GHz third-order Butterworth low Pass filter, its S-parameter frequency response graph.

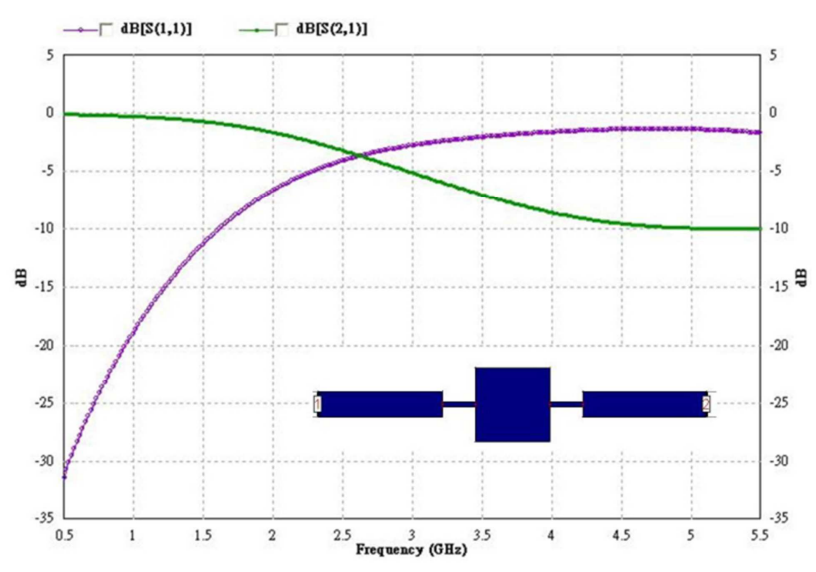

Figure 2. A typical step impedance $2.5 \mathrm{GHz}$ third-order Butterworth microstrip line low-pass filter S-parameter frequency response diagram and its microstrip circuit structure (lower right illustration)

It can be seen from the frequency response diagram in Figure 2 that the performance of the traditional step impedance microstrip line low-pass filter is not ideal. The value of S21 in the stop band is too large, and the value of S11 in the pass band is not small enough.

We changed the microstrip line low-pass filter to use an open-circuited stub and added a semi lump type design the simulated operating frequency of $2.5 \mathrm{GHz}$ third-order Butterworth low pass filter. The S-parameter frequency response diagram is shown in Figure 3. The lower illustration in Figure 3 shows the microstrip circuit structure of a semi-lumped $2.5 \mathrm{GHz}$ third-order Butterworth microstrip line low-pass filter. 


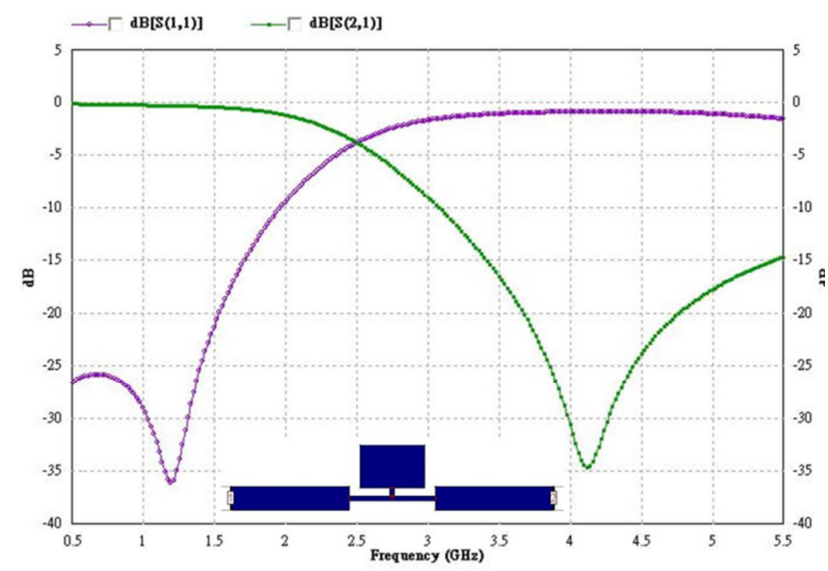

Figure 3. Semi-lumped element 2.5GHz third-order Butterworth microstrip line low-pass filter S-parameter frequency response diagram and its microstrip circuit structure (lower middle illustration)

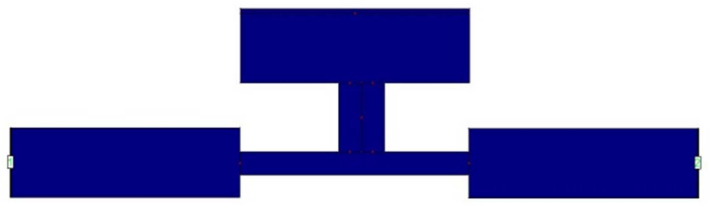

(a)

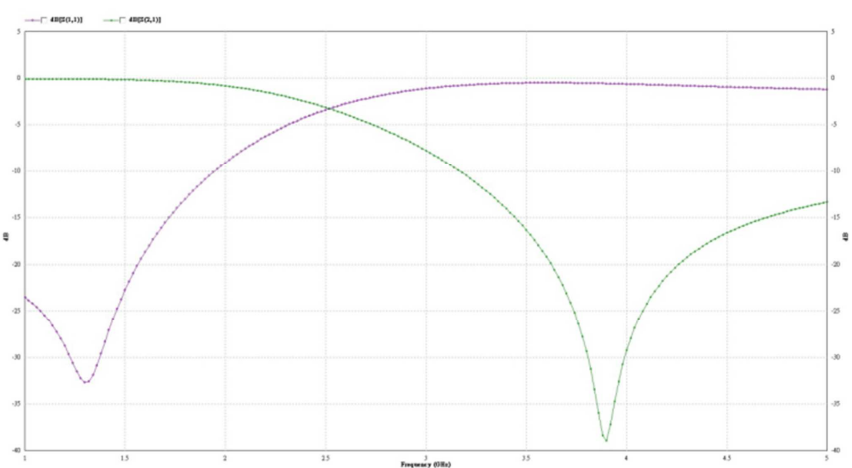

(b)

Figure 4. Microstrip line semi-lumped element low-pass filter (a) Microstrip line circuit structure diagram, (b) Simulated S-parameter frequency response diagram

\section{Results and Discussion}

Because an LC resonance zero point is introduced at the stop band in the low-pass filter of the traditional semi-lumped element microstrip line. Therefore, in theory, it will have better stop-band characteristics than the step impedance or open-circuited stub microstrip line low-pass filter, and the transition bandwidth will be steeper. 
We actually designed a low-pass filter with a half-strip lumped component microstrip line. The simulated frequency response characteristics are not ideal and need further improvement.

Figure 4 shows the microstrip circuit structure diagram (Figure 4 (a)) and the simulated S-parameter frequency response diagram of the semi-lumped component microstrip line low-pass filter (Figure 4 (b)).

The frequency response diagram of the S-parameter simulated by the electromagnetic simulation software in Figure 4 (b), where the purple line is the reflection coefficient s11 at the input end and the green line is the forward transmission coefficient $\mathrm{s} 21$. Its $-3 \mathrm{~dB}$ bandwidth is $2.5 \mathrm{GHz}$. In this figure, it is also at the junction of the purple and green lines. Below $2.5 \mathrm{GHz}$, the forward transmission coefficient is the largest, and the reflection coefficient at the input end of $2.5 \mathrm{~Hz}$ to $5 \mathrm{~Hz}$ is the largest. $1.3 \mathrm{GHz}$ is the best signal passing, and $3.9 \mathrm{GHz}$ is the least signal passing. Figure 5 shows a photo of the actual implementation of the microstrip line semi-lumped element filter of Figure 4.

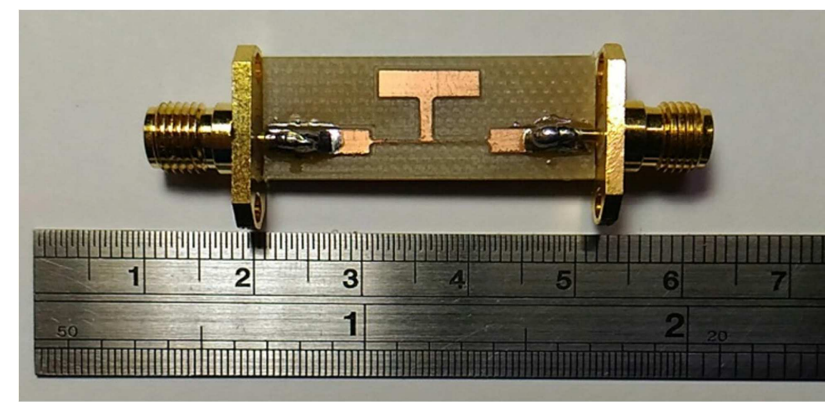

Figure 5. The actual photo of the implementation of the microstrip line semi-lumped element filter in Figure 4

However, the transition bandwidth of the low-pass filter of the semi-lumped element is still not steep enough, and the stopband characteristic is still not good enough. Therefore, we will introduce the original traditional microstrip line semi-lumped LPF into two quarter-wavelength open-circuited stubs with different components, using a quarter Band rejection filtering characteristics of open short-wavelength branches. To improve the original semi-lumped element low-pass filter transition bandwidth is not steep enough, poor stopband characteristics and other issues. When we need a wider band rejection frequency ratio, basically the characteristic impedance of the open-circuited stubs is relatively small, that is, a thicker wire, so it will increase a lot of area. At the same time, the combination of the two circuit structures also needs to reconsider the problem of impedance matching.

Figure 6 introduces the open-circuited stubs with different quarter wavelength open circuit. Fig. 6 (a) is a microstrip line circuit structure diagram, and Fig. 6 (b) is a simulation s-parameter frequency response diagram.

The frequency response graph of the $\mathrm{S}$ parameter simulated by the electromagnetic simulation software in Fig. 6 (b), where the purple line is the reflection coefficient at the input end and the green line is the forward transmission coefficient $\mathrm{s} 21$.

Its $-3 \mathrm{~dB}$ bandwidth is $2.5 \mathrm{GHz}$. In this figure, it is also at the junction of the purple and green lines. Below $2.5 \mathrm{GHz}$, the forward transmission coefficient is the largest, and the reflection coefficient at the input end of $2.5 \mathrm{~Hz}$ to $5 \mathrm{~Hz}$ is the largest. The reflection coefficient of the input terminal gradually increases below $2.5 \mathrm{GHz}$, but the signals below $1.9 \mathrm{GHz}$ are below $-30 \mathrm{~dB}$, which is an excellent passband. The reflection coefficient from $2.7 \mathrm{GHz}$ to $4.1 \mathrm{GHz}$ is the largest and the forward transmission coefficient is the smallest, which is an excellent stop band. 
Comparing Fig. 4 (b) and Fig. 6 (b), it can be seen that this low-pass filter with a bifurcated quarterwavelength an open-circuited stub microstrip line has a passband at $2 \mathrm{GHz}$ to $2.5 \mathrm{GHz}$ and $2.5 \mathrm{GHz}$ to $3.5 \mathrm{GHz}$ Stop the band and add a branch of the quarter-wavelength open circuit. Figure 6 (b) is much better than the original figure 4 (b).

In general, the reflection loss in the passband and the insertion loss characteristics of the stopband, the addition of the branched quarter-wavelength open-circuited stubs is better than the original figure 4 (b). The filter characteristics of open-circuited stubs wavelength have been improved.

Fig. 7 is a physical photograph of the implementation of the open-circuited stubs filter of the quarterwavelength of the two components of the microstrip line of Fig. 6 with different components.

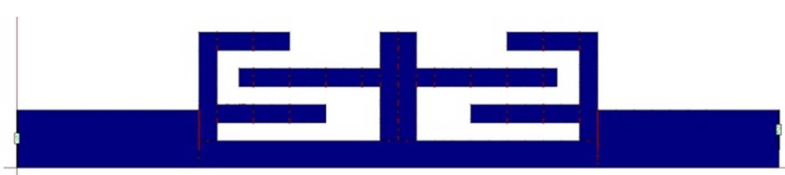

(a)

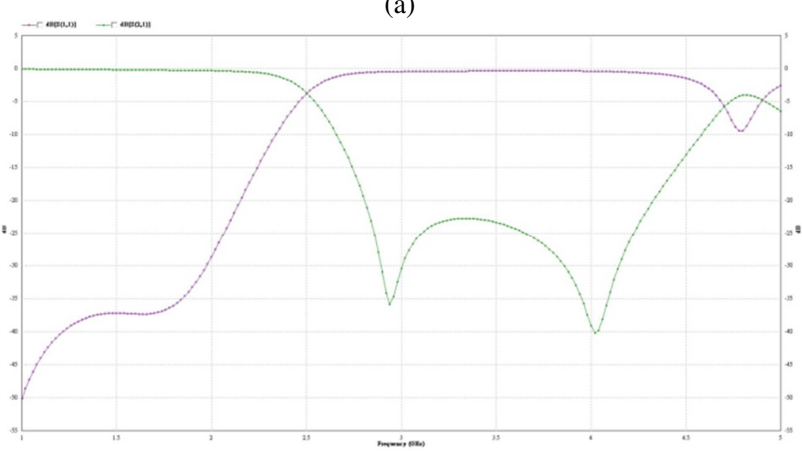

(b)

Figure 6. Microstrip line with two components of different quarter-wavelength open-circuited stubs filters (a) Microstrip line circuit structure diagram, (b) Simulated S-parameter frequency response diagram

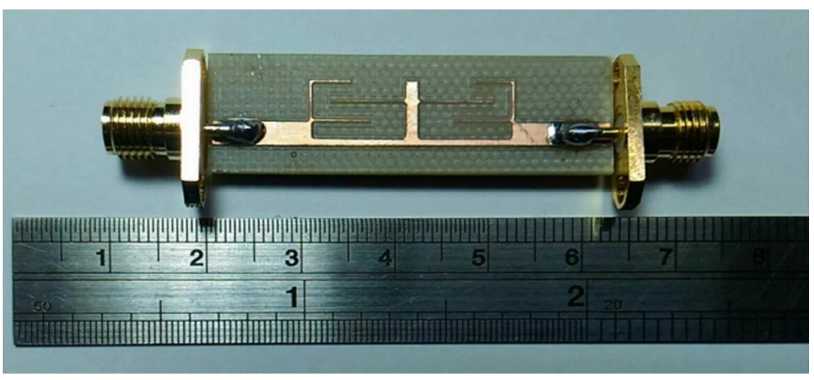

Figure 7. The actual photo of the implementation of two quarter-wavelength open-circuited stubs filters with different components in the microstrip for Figure 6 


\section{Conclusion}

The quarter-wavelength bifurcated quarter-wavelength open-circuited stub microstrip line low-pass filter designed by our people is a low-pass filter operating at $2.5 \mathrm{GHz}$. Compared with the traditional semi-lumped element microstrip line low-pass filter, its frequency response characteristics are particularly obvious in improving the transition bandwidth. Passband and stopband characteristics have also been improved.

That is, the proper use of quarter-wavelength open-circuited stub can effectively improve the insertion loss and reflection loss characteristics of the passband and stopband of the low-pass filter.

\section{Acknowledgements}

This work is supported by the Industry-University Cooperation Plan of Fareast University in Taiwan, Republic of China (grant 10803-08-36-002).

\section{References}

Technology foresight, New Communications, No.132, February 2012.

Jia-Sheng Hong and M.J. Lancaster, "Microstrip Filters for RF/MicrowaveApplication",2001.

David H. Schrader, "Microstrip Circuit Analysis", 1995.

Shi-Hong Li, Microwave Engineering, Wunan Books Co.,Ltd., 2011.

Di-Wen Yuan, and Yue-HuaWang, High frequency communication circuit design, Gaoli Book Co.,Ltd., 2015.

Xiaolei Sun, S. W. Cheung, and T. I. Yuk, "Coplanar waveguide-fed dual-band antenna for wireledd local area network application," Microwave and Optical Technology Letters, Vol. 54, No. 6, June 2012

Chen Wang, Ze-Hong Tan, Ping Xu, and Sheng Li, "Compact ultra-wideband coplanar waveguide fed fan-shaped antenna with bandnotched characteristic," Microwave and Optical Technology Letters, Vol. 55, No. 1, Jan. 2013.

Jang-Yeol Kim, Nam Kim, Seungwoo Lee, and Byoung-Cheol Oh, "Triple band-notched UWB monopole antenna with two resonator structures,"Microwave and Optical Technology Letters, Vol.55, No. 1, Jan. 2013.

Joong-Han Yoon, Young-Chul Rhee, and Yeon-Kil Jang, "A study on the rectangular ring, openended monopole antenna with a vertical strip for WLAN dual-band operations," Microwave and Optical Technology Letters, Vol. 55, No. 3, Mar. 2013.

Nasser Ojaroudi and Mohammad Ojaroudi, "A novel design of reconfigurable small monopole antenna with switchable band notch and multiresonance functions for UWB applications," Microwave and Optical Technology Letters, Vol.55, No. 3, Mar. 2013.

EP-2006 engraving machine operation manual, 2013. 